\title{
Lithium Production in White Dwarf Mergers
}

\author{
Richard Longland ${ }^{*}{ }^{a b}$ Pablo Lorén-Aguilar, ${ }^{c b}$ Jordi José, ${ }^{a b}$ Enrique García-Berro, ${ }^{c b}$ \\ and Leandro Althaus ${ }^{d}$ \\ ${ }^{a}$ Departament de Física i Enginyeria Nuclear, EUETIB, Universitat Politècnica de Catalunya \\ C/ Comte d'Urgell 187, E-08036 Barcelona, Spain \\ ${ }^{b}$ Institut d'Estudis Espacials de Catalunya (IEEC) \\ Ed. Nexus-201, C/ Gran Capità 2-4, E-08034 Barcelona, Spain \\ ${ }^{c}$ Departament de Física Aplicada, Universitat Politècnica de Catalunya, \\ C/Esteve Terrades, 5,E-08860 Castelldefels, Spain \\ ${ }^{d}$ Facultad de Ciencias Astronómicas y Geofísicas, Universidad Nacional de La Plata \\ Paseo del Bosque s/n, (1900) La Plata, Argentina \\ E-mail: richard.longlandeupc.edu
}

\begin{abstract}
White dwarf mergers are an interesting phenomenon that can explain the formation of some astrophysical objects that cannot be attributed to standard stellar evolution. Amongst these phenomena are $\mathrm{R}$ Coronae Borealis stars, for which the origin of their peculiar surface abundances has eluded us for over 200 years. Mergers of helium and carbon-oxygen white dwarfs can account for some of their surface abundances. Recently, however, studies have pointed to the existence of lithium in these stars as evidence that this merger scenario cannot explain their formation. In this work, we summarise how lithium can, indeed, be produced by mergers of white dwarfs. This is achieved by carefully accounting for the white dwarf abundances with full evolutionary models, and mapping them to hydrodynamic models and nucleosynthesis post-processing. We also investigate the sensitivity of this production on the dynamical properties of the merger.
\end{abstract}

XII International Symposium on Nuclei in the Cosmos

August 5-12, 2012

Cairns, Australia

\footnotetext{
* Speaker.
} 


\section{Introduction}

Mergers of white dwarf stars are a fairly common event in our galaxy. Approximately two in every three new-born stars are estimated to be born in a binary system. Of these binary systems, approximately 5 are expected to result in a double white dwarf system every 100 years [四]. Their investigation is therefore an important subject of research leading to a number of possible scenarios []. $]$. One such scenario is the merging of a helium white dwarf with one comprising of carbon and oxygen. This scenario has been proposed as the mechanism for producing $\mathrm{R}$ Coronae Borealis ( $\mathrm{RCrB})$ stars.

$\mathrm{RCrB}$ stars are hydrogen poor, helium rich stars that have peculiar chemical signatures. They are known to be overabundant (with respect to solar abundances) in carbon, oxygen, fluorine, and lithium. For a more thorough discussion of the surface abundances of RCrB stars, the reader is referred to Ref. [B] and references therein. Recent progress [B], 团] showed that fluorine and oxygen can be synthesised successfully in white dwarf mergers, adding credibility to the white dwarf merger scenario for their formation. However, other studies [ [l] point to the presence of lithium in these stars as evidence against this theory. They argue that any lithium present in the white dwarfs prior to the merging event would be efficiently destroyed by the high temperatures reached during the merger. This argument neglects the possibility that lithium can be produced from existing material in the white dwarfs during the merger.

The key to understanding lithium production in white dwarf mergers comes from considering the origins of the white dwarfs. The white dwarfs originate from stars that have undergone rich and complex evolution. The ashes of that evolution are not fully expelled during the common envelope mass loss stages preceding the binary white dwarf system. It is from these ashes that lithium can be synthesised.

\section{Nucleosynthesis prior to the merger}

In order to produce a white dwarf binary system consisting of a $0.8 \mathrm{M}_{\odot}$ carbon-oxygen white dwarf and a helium white dwarf with $0.4 \mathrm{M}_{\odot}$, a binary progenitor system of stars with about $2.5 \mathrm{M}_{\odot}$ and $1 \mathrm{M}_{\odot}$ is required. Because of its higher mass, the $2.5 \mathrm{M}_{\odot}$ star will evolve before the low mass star leaves the main sequence. This star evolves up the red giant branch, through the first and second dredge-up events, and into the AGB phase [圆. During the AGB phase, the star undergoes hydrogen and helium shell burning, before finally losing its envelope in a common envelope stage (see Ref. [ $[$ ] for a good overview of this binary evolution). Angular momentum losses during this stage serve to bring the two stars closer together. The final result of such an evolutionary track is a main sequence star of $1 \mathrm{M}_{\odot}$ in a binary system with a carbon-oxygen white dwarf. The carbon-oxygen white dwarf will contain thin shells of partially burned hydrogen and helium.

The $1 \mathrm{M}_{\odot}$ star will then evolve in a similar manner. However, in this case, the star loses its envelope earlier than if it were a bare star because of its proximity to the other white dwarf. The star will therefore lose its envelope while on the red giant branch, thus producing a helium white dwarf remnant. This star contains a thin shell of partially burned hydrogen-rich material. 
Material left in these partially burned, hydrogen-rich buffer regions has undergone nuclear processing. Before a star loses its envelope, these regions are buried deep within the stars at high temperatures and densities, where hydrogen burning proceeds primarily through the $\mathrm{CNO}$ cycle, but also though the pp-chain. By solving the partial differential equations determining the evolution of pp-chain material (see Refs. [ $[\mathbb{0}, \mathbf{Q}]$ for more details), we find that ${ }^{3} \mathrm{He}$ reaches an equilibrium abundance of

$$
\left({ }^{3} \mathrm{He}\right)_{e}=\frac{1}{2\langle\sigma v\rangle_{33}}\left[-\left({ }^{4} \mathrm{He}\right)\langle\sigma v\rangle_{34}+\sqrt{2(\mathrm{H})^{2}\langle\sigma v\rangle_{p p}\langle\sigma v\rangle_{33}+\left({ }^{4} \mathrm{He}\right)^{2}\langle\sigma v\rangle_{34}^{2}}\right] .
$$

In a $1 \mathrm{M}_{\odot}$ red giant, hydrogen shell burning occurs at 10-20 MK. Using Eq. (D. defined as consisting of $50 \%$ hydrogen and $50 \%$ helium, we find that a ${ }^{3} \mathrm{He}$ equilibrium abundance of $\left({ }^{3} \mathrm{He}\right)_{e} \approx 1 \times 10^{-5}$ is reached in $10^{5}$ years. This timescale is significantly less than the star's lifetime on the red giant branch. It follows that once the star's envelope has been lost, revealing the partially burned material below, a thin buffer of material enriched in ${ }^{3} \mathrm{He}$ will remain.

\section{Nucleosynthesis during the merger}

Following angular momentum loss by gravitational wave emission, tidal forces serve to bring the two white dwarfs close together. Once the helium white dwarf starts to undergo mass transfer, it is rapidly disrupted and accreted onto the carbon-oxygen white dwarf [ए]]. During this process, material from the helium white dwarf undergoes rapid heating, followed by cooling as it rebounds back to larger orbits. We showed in Sec. \ that the buffer material is enriched in ${ }^{3} \mathrm{He}$. Let us now consider nucleosynthesis in this material as it faces these extreme conditions.

To investigate ${ }^{7} \mathrm{Li}$ production during the merger, we first assume the same buffer region as discussed previously, i.e., $X(\mathrm{H})=0.5, X\left({ }^{4} \mathrm{He}\right)=0.5$, and $X\left({ }^{3} \mathrm{He}\right)=1 \times 10^{-5}$. A parametrised profile is used to investigate the sensitivity of ${ }^{7} \mathrm{Li}$ synthesis on the timescales and temperatures reached in the merger. The profile is defined by (i) the maximum temperature reached, (ii) the time taken for the material to be heated from $T=1 \mathrm{MK}$ to the maximum temperature, (iii) the time spent at the maximum temperature, and (iv) the time required for the material to cool back to $T=1 \mathrm{MK}$. The heating and cooling episodes are assumed to be adiabatic. We find that the most ${ }^{7} \mathrm{Li}$ is synthesised for fast heating and cooling profiles with maximum temperatures of $350 \mathrm{MK}$. In reality, we do expect the heating and peak timescales to be short during the merger, but the cooling timescale could. Therefore, the sensitivity of ${ }^{7} \mathrm{Li}$ production to the maximum temperature reached and the cooling time is presented in Fig. $\mathrm{W}$ (assuming a rise time and peak times of $1 \mathrm{~s}$ ).

As temperature rises in the material, ${ }^{3} \mathrm{He}$ and ${ }^{4} \mathrm{He}$ react to produce ${ }^{7} \mathrm{Be}$. This ${ }^{7} \mathrm{Be}$ can undergo electron capture with a half-life of about 53 days to produce ${ }^{7} \mathrm{Li}$. However, further reactions take place. To explore the possibilities, consider Fig. 四, which can be divided into three temperature regions, (i) low temperatures below $\mathrm{T}=100 \mathrm{MK}$, (ii) medium temperatures between $100 \mathrm{MK}$ and $400 \mathrm{MK}$, and (iii) high temperatures above $400 \mathrm{MK}$. In the low temperature regime, ${ }^{7} \mathrm{Be}$ is produced but destruction through further proton and helium captures is inefficient. At these temperatures, moderate ${ }^{7} \mathrm{Be}$ levels can be reached that are relatively insensitive to the time-scales of the merger. Once temperatures exceed $100 \mathrm{MK}$, the ${ }^{7} \mathrm{Be}(\mathrm{p}, \gamma)^{8} \mathrm{~B}$ reaction destroys ${ }^{7} \mathrm{Be}$ to produce ${ }^{8} \mathrm{Be}$, which subsequently decays into two $\alpha$-particles. As temperatures rise further towards $300 \mathrm{MK}$, however, 


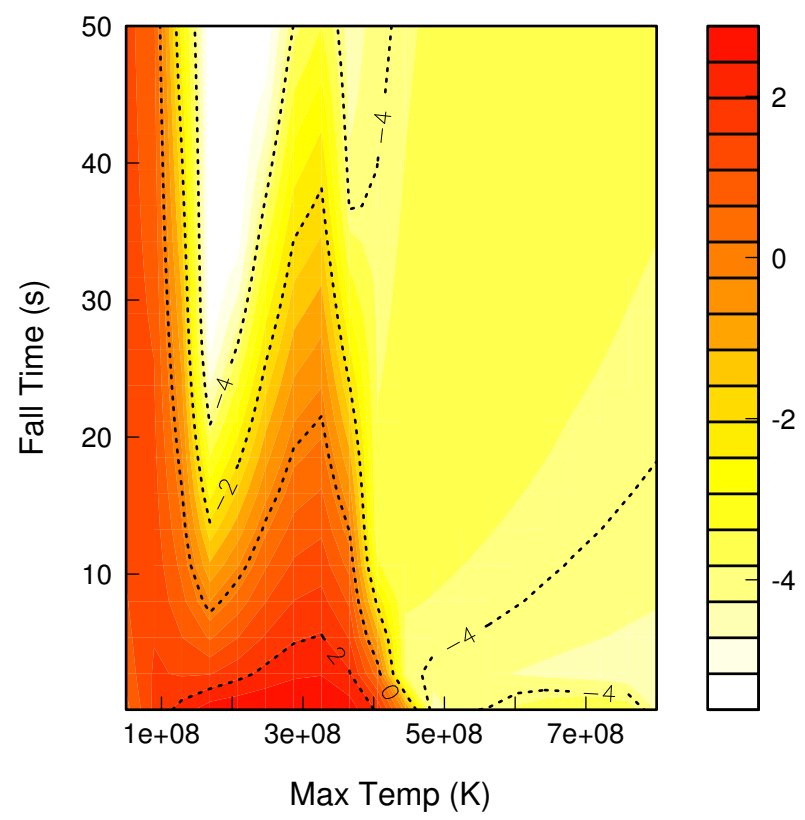

Figure 1: ${ }^{7} \mathrm{Li}$ production in buffer material as a function of both maximum temperature reached in the merger, and the fall time (in seconds) for the material to cool to $1 \mathrm{MK}$. The colour scale corresponds to the abundance with respect to solar in logarithmic units. For example, a value of 0 (orange) corresponds to solar values of ${ }^{7} \mathrm{Li}$, while a value of 2 (red) corresponds to a factor of 100 more than solar lithium abundance.

this destruction is ameliorated by the reverse reaction, ${ }^{8} \mathrm{~B}(\gamma, \mathrm{p})^{7} \mathrm{Be}$, which establishes an equilibrium of ${ }^{7} \mathrm{Be}$ and ${ }^{8} \mathrm{~B}$ determined by the ratio of their reaction rates. At these temperatures, the ${ }^{10} \mathrm{~B}(\mathrm{p}, \gamma){ }^{9} \mathrm{C}$ reaction reduces the amount of ${ }^{7} \mathrm{Be}$ produced in slower profiles, as is evident from the abundance drop as a function of fall time at about $300 \mathrm{MK}$ in Fig. 四. Finally, as temperatures rise even higher (above $\mathrm{T} \approx 400 \mathrm{MK}$ ), the ${ }^{7} \mathrm{Be}(\alpha, \gamma){ }^{11} \mathrm{C}$ reaction becomes efficient, thereby destroying ${ }^{7} \mathrm{Be}$ efficiently with no path for recovery.

\section{Full nucleosynthesis models}

Sections $\square$ and $\mathrm{B}$ described the theoretical mechanism for lithium production during white dwarf mergers. This mechanism can also be tested directly in hydrodynamical models. By mapping white dwarf evolutionary models [W], [2] to Smoothed Particle Hydrodynamics (SPH) models of white dwarf mergers [지], nucleosynthesis in the white dwarf buffer region can be taken into account.

The white dwarf models are first used to evolve $2.5 \mathrm{M}_{\odot}$ and $1 \mathrm{M}_{\odot}$ stars from the main sequence to the white dwarf stage, thus obtaining abundances as a function of radius. An enhanced mass loss rate is used for the $1 \mathrm{M}_{\odot}$ star to approximate the common envelope stage (and therefore mass loss) on the red giant branch as discussed in Sec. [3. SPH models using 300000 particles are then used with a limited nuclear network (sufficient to reproduce energy production) to evolve a binary system of a $0.8 \mathrm{M}_{\odot}$ carbon-oxygen white dwarf and a $0.4 \mathrm{M}_{\odot}$ helium white dwarf. 
Once the SPH models have been computed, a selection 10000 of tracer particles is taken. These tracer particles are chosen to represent particles that will end their evolution in the atmosphere and accretion disk of the merger product. For each tracer particle, initial abundances are assigned according to their radius from the detailed white dwarf models, and nucleosynthesis subsequently followed using the tracer particle temperature-density profiles. Following this evolution, the final abundances in our selection of tracer particles is mass-averaged as a function of radius.

The final lithium abundances as a function of radius in the final object is shown in Fig. $\square$. It is clear from this figure that by carefully considering the partially burned hydrogen buffers obtained from white dwarf models, the lithium abundance in the accretion disk of a white dwarf merger product can be enhanced to high values. These values agree remarkably well with the lithium abundances observed in $4 \mathrm{RCrB}$ stars [ []] , which are approximately two orders of magnitude higher than those present in the solar system. Figure $\square$ also illustrates that lithium production is a primary process, independent of metalicity.

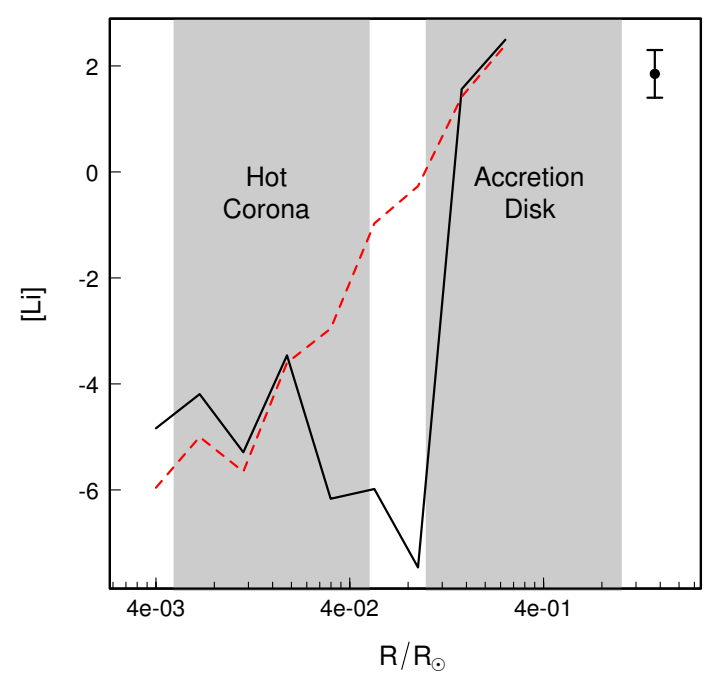

Figure 2: Mass averaged lithium abundance as a function of radius in the final merger product. The abundance is plotted with respect to solar abundance on a logarithmic scale. The solid black and dashed red lines correspond to the abundances obtained from solar metalicity, and $z=1 \times 10^{-5}$, respectively. The vertical bars represent the regions in the final object corresponding to the hot corona close to the dense, central carbon-oxygen white dwarf, and the more diffuse accretion disk surrounding the object. The data point represents the range of observed lithium abundances in RCrB stars [3]].

\section{Conclusions}

In this poster, we showed that lithium can, indeed, be produced in the merging of white dwarf stars. During the evolution of stars prior to the white dwarf stage, they undergo a number of nuclear burning phases, leading to regions of partially burned material. As these stars lose their envelopes to winds in the common envelope stage of their evolution, this partially burned material is not completely lost and remains on the surface of the white dwarf. This material is enriched in ${ }^{3} \mathrm{He}$. 
When the binary white dwarf system merges, this ${ }^{3} \mathrm{He}$ rich material undergoes rapid heating and cooling, in which ${ }^{7} \mathrm{Be}$ (and thus, ${ }^{7} \mathrm{Li}$ ) can be produced.

It was previously thought [5] that lithium in $\mathrm{RCrB}$ stars indicated that they could not be formed in the merger of white dwarfs. Here we show that this evidence can no longer be used to rule out this scenario. However, the lithium is predicted in our models to be confined to the outer regions of the final merger product (see, also, Ref. [ [ $]$ ] ). This could provide a valuable test of the origin of $\mathrm{RCrB}$ stars if the location of lithium in these systems can be identified.

\section{References}

[1] G. Nelemans, L. R. Yungelson, S. F. Portegies Zwart, and F. Verbunt, Population synthesis for double white dwarfs . I. Close detached systems, Astron. Astrophys. 365 (2001) 491-507, astro-ph/0010457].

[2] I. Iben, Jr., A. V. Tutukov, and L. R. Yungelson, Helium and Carbon-Oxygen White Dwarfs in Close Binaries, ApJ 475 (Jan., 1997) 291.

[3] C. S. Jeffery, A. I. Karakas, and H. Saio, Double white dwarf mergers and elemental surface abundances in extreme helium and R Coronae Borealis stars, MNRAS 414 (2011) 3599-3616, astro-ph/1103.1556.

[4] R. Longland, P. Lorén-Aguilar, J. José, E. García-Berro, L. G. Althaus, and J. Isern, Nucleosynthesis during the Merger of White Dwarfs and the Origin of R Coronae Borealis Stars, Astrophys. J. 737 (2011) L34, astro-ph/1107.2233].

[5] G. C. Clayton, B. E. K. Sugerman, S. A. Stanford, B. A. Whitney, J. Honor, B. Babler, et al., The Circumstellar Environment of R Coronae Borealis: White Dwarf Merger or Final-helium-shell Flash?, Astrophys. J. 743 (2011) 44, [astro-ph/1110.3235].

[6] H. Habing and H. Olofsson, Asymptotic giant branch stars. Springer, 2004.

[7] I. Iben, Jr. and A. V. Tutukov, Supernovae of type I as end products of the evolution of binaries with components of moderate initial mass (M not greater than about 9 solar masses), Astro. Phys. J. Supp. 54 (1984) 335-372.

[8] R. Longland, P. Lorén-Aguilar, J. José, E. García-Berro, and L. G. Althaus, Lithium production in the merging of white dwarf stars, Astron. Astrophys. 542 (2012) A117, [ast ro-ph/1205.2538].

[9] C. Iliadis, Nuclear physics of stars. Wiley-VCH, 2007.

[10] P. Lorén-Aguilar, J. Isern, and E. García-Berro, High-resolution smoothed particle hydrodynamics simulations of the merger of binary white dwarfs, Astron. Astrophys. 500 (2009) 1193-1205.

[11] I. Renedo, L. G. Althaus, M. M. Miller Bertolami, A. D. Romero, A. H. Córsico, R. D. Rohrmann, and E. García-Berro, New Cooling Sequences for Old White Dwarfs, Astrophys. J. 717 (2010) 183-195, [astro-ph/1005.2170].

[12] L. G. Althaus, E. García-Berro, I. Renedo, J. Isern, A. H. Córsico, and R. D. Rohrmann, Evolution of White Dwarf Stars with High-metallicity Progenitors: The Role of ${ }^{22}$ Ne Diffusion, Astrophys. J. 719 (2010) 612-621, [astro-ph/1006.4170].

[13] P. Lorén-Aguilar, J. Isern, and E. García-Berro, Smoothed particle hydrodynamics simulations of white dwarf collisions and close encounters, MNRAS 406 (2010) 2749-2763,

astro-ph/1004.4783. 